\title{
A generalized thermodynamic approach to microrheological modelling
}

\author{
R.J.J. JONGSCHAAP \\ Twente University of Technology, Department of Applied Physics, Enschede, The Netherlands
}

Received 4 July 1990; accepted in revised form 3 November 1990

\begin{abstract}
A generalized thermodynamic theory is presented which may be applied to microrheological models. The purpose of this theory is to offer a simple framework for many types of modelling at various levels of description.

The essential elements of our approach are: a specification of the subsystem in which the reversible storage of energy takes place, the way of coupling of this subsystem to the environment and a proper definition of reversible and irreversible variables. The resulting set of equations, containing a stress tensor expression a microscopic evolution equation and a microscopic equation of state are expressed in a matrix form.

Some applications of the theory to well known micro-rheological models are discussed and directions for further developments are indicated.
\end{abstract}

\section{Introduction}

Microrheological models are used in order to study the relation between the flow properties of materials and their microstructure. Since there exist many different types of modelling, a large number of apparently different approaches are known.

In addition there is the possibility to treat a single system at various levels of description. We will speak of a low level of description if many details of the microstructure are taken into account and of a high level of description in the case of a more global representation. A description in terms of the coordinates and momenta of the individual particles, for instance, is at a low level and a description based upon configurational distribution functions at a higher level of description.

The purpose of the present paper is to show how in the wide variety of types of modelling a considerable unification may be achieved, by using some kind of thermodynamic approach. In the next section a general outline of this approach will be given and after that some applications will be discussed.

\section{Theory}

In the flow or deformation of a material the mechanical power, supplied to it is partly stored and partly dissipated. In general a certain subsystem may be defined in which the storage of power takes place. The reversible storage of energy under isothermal conditions is determined then by the free energy function or functional $A=A(\Phi)$. Here, $\Phi$ is a (set of) state variable(s) or function(s). This set should be complete in the 
sense that - also under non-equilibrium conditions - the free energy is fully determined by $\Phi$.

We also introduce a (set of) thermodynamic force(s):

$$
\Pi=\frac{\delta A}{\delta \Phi}
$$

(The type of the derivative in this expression depends upon the character of the variable $\Phi$.) The rate of change of free energy then becomes

$$
\dot{A}=\Pi \cdot \dot{\Phi}
$$

This is the reversible storage of mechanical power in the selected subsystem.

On the other hand the power supply to the system is given by

$$
W=\Sigma \cdot \dot{\Gamma}
$$

in which $\Sigma$ is a (set of) external force(s) and $\dot{\Gamma}$ a (set of) rates of displacement variables. The type of multiplication (contraction) in (2) may differ from the one used in (3). In this section however we use the same notation in both cases. The relations between the force and displacement variables depends upon specific properties of the system. Some general conditions upon these relations will be discussed below.

In order to illustrate the concepts introduced so far, consider the mechanical model shown in Fig. 1. In that case the free energy function becomes $A=\frac{1}{2} k \Phi^{2}$ in which $k$ is the spring-modulus and $\Pi=k \Phi$ is the reversible force. The relation between the

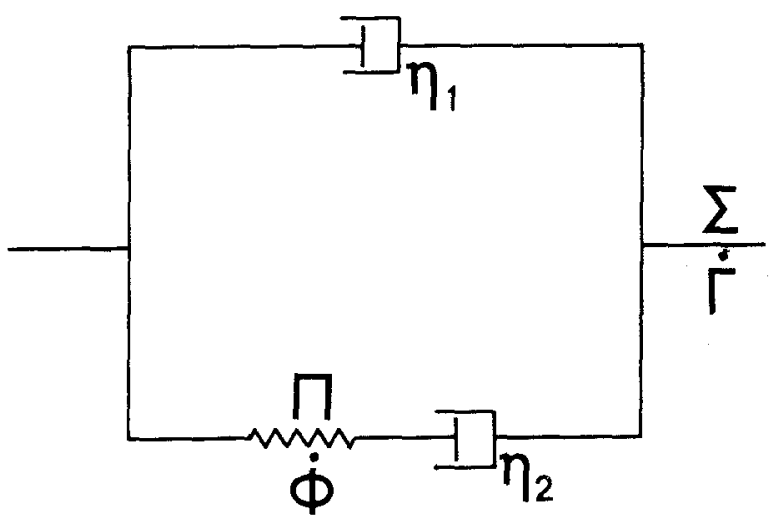

Fig. 1. Mechanical model, illustrating the differences between the variables $\Sigma$ and $\dot{\Gamma}$, by which the power supply taken place and the variables $\Pi$ and $\dot{\Phi}$ occurring in the expression (2.2) for the reversible rate of storage of energy. The coupling between $\Sigma, \Pi, \dot{\Gamma}$ and $\dot{\Phi}$ is given by the relations $(2.10)$. 
variables $\Sigma, \Pi, \dot{\Gamma}$ and $\dot{\Phi}$ becomes

$$
\begin{aligned}
& \dot{\Gamma}=\dot{\Phi}+\frac{1}{\eta_{2}} \Pi, \\
& \Sigma=\Pi+\eta_{1} \dot{\Gamma} .
\end{aligned}
$$

In these expressions, $\eta_{1}$ and $\eta_{2}$ are the viscosities of the dashpots, shown in Fig. 1. It is important to note that the structure of these equations reflects the way in which the substructure in which storage takes place (the spring) is connected to the environment.

Returning again to the general case, we will discuss now the general structure of equations of the type (2.4) and (2.5). To that end we first note that by (2.2) and (2.3) the rate of dissipation $\Delta=W-\dot{A}$ is given by

$$
\Delta=\Sigma \cdot \dot{\Gamma}-\Pi \cdot \dot{\Phi}
$$

In order to distinguish 'reversible' and 'dissipative' parts of the variables in this expression we consider the parity of variables under the transformation $\dot{\Gamma} \rightarrow-\dot{\Gamma}$. This transformation will be called a 'macroscopic time reversal'. A variable $\Psi$ may be decomposed as

$$
\Psi=\Psi^{+}+\Psi^{-} \quad \text { with } \Psi^{+}=\frac{1}{2}(\Psi(\dot{\Gamma})+\Psi(-\dot{\Gamma}))
$$

and

$$
\Psi^{-}=\frac{1}{2}(\Psi(\dot{\Gamma})-\Psi(-\dot{\Gamma}))
$$

By definition we have $\dot{\Gamma}=\dot{\Gamma}^{-}$and since $\Pi$ is a state variable: $\Pi=\Pi^{+}$. Furthermore $\Delta=\Delta^{+}$, since $\Delta \geqslant 0$, by the second law of thermodynamics. From the dissipation expression (2.6) we now obtain

$$
\begin{aligned}
& \Delta^{+}=\Sigma^{-} \cdot \dot{\Gamma}-\Pi \cdot \dot{\Phi}^{+} \geqslant 0, \\
& \Delta^{-}=\Sigma^{+} \cdot \dot{\Gamma}-\Pi \cdot \dot{\Phi}^{-}=0 .
\end{aligned}
$$

The quantities $\Sigma^{-}$and $\dot{\Phi}^{+}$, occurring in (2.7) will be called 'dissipative' and denoted by $\Sigma^{D}$ and $\dot{\Phi}^{D}$ and the quantities $\Sigma^{+}$and $\dot{\Phi}^{-}$in (2.8), 'reversible' and denoted by $\Sigma^{R}$ and $\dot{\Phi}^{R}$, respectively.

By considering the parity of the variables in (2.7) and (2.8) it can be shown (see Appendix A) which couplings between them are admissible. We restrict ourselves to the case that $\Sigma$ and $\Pi$ depend linear on $\dot{\Gamma}$. In that case the admissible couplings may be 
summarized in the following matrix expressions:

$$
\left[\begin{array}{c}
\Sigma^{R} \\
\dot{\Phi}^{R}
\end{array}\right]=\left[\begin{array}{rr}
0 & -\Lambda^{T} \\
\Lambda & 0
\end{array}\right]\left[\begin{array}{r}
\dot{\Gamma}^{R} \\
-\Pi^{R}
\end{array}\right] ; \quad\left[\begin{array}{c}
\Sigma^{D} \\
\dot{\Phi}^{D}
\end{array}\right]=\left[\begin{array}{ll}
\eta & 0 \\
0 & \beta
\end{array}\right]\left[\begin{array}{r}
\dot{\Gamma} \\
-\Pi
\end{array}\right] .
$$

In the case of tensorial variables the appropriate contractions should be taken into account. The transposed $\Lambda^{T}$ is defined by $(\Lambda * Z) \cdot Y=\left(\Lambda^{T} \cdot Y\right) * Z$ in which $*$ and . denote the appropriate contractions. In the case that the variables are functions the matrix elements should be considered as operators. By addition of the two expressions (2.9) we get

$$
\left[\begin{array}{l}
\Sigma \\
\dot{\Phi}
\end{array}\right]=\left[\begin{array}{rr}
\eta & -\Lambda^{T} \\
\Lambda & \beta
\end{array}\right]\left[\begin{array}{r}
\dot{\Gamma} \\
-\eta
\end{array}\right] .
$$

This result may be considered as the set of rate equations, corresponding to the dissipation expression (2.6) according to the usual procedures of nonequilibrium thermodynamics [7]. Note that in (2.10) the Onsager-Casimir reciprocal relations are obeyed. From (2.1) and (2.9) we obtain

$$
\Sigma^{R}=\frac{\delta \dot{\Phi}^{R}}{\delta \dot{\Gamma}} \cdot \frac{\delta A}{\delta \Phi}
$$

This corresponds to a general expression for the reversible part of the stress tensor originally obtained by Grmela [5] in a general bracket formulation of convection and diffusion equations.

The equations (2.4) and (2.5) corresponding to the example of Fig. 1, displayed in the form (2.10) read

$$
\left[\begin{array}{l}
\Sigma \\
\dot{\Phi}
\end{array}\right]=\left[\begin{array}{cc}
\eta_{1} & -1 \\
1 & \eta_{2}^{-1}
\end{array}\right]\left[\begin{array}{r}
\dot{\Gamma} \\
-\Pi
\end{array}\right]
$$

so in that case we have $\Lambda=1$.

A mechanical model, corresponding to the case with $\Lambda \neq 1$ is shown in Fig. 2 . Because of its shape we will call this model the 'Triangle Model'. It may be considered as a symbolic representation of our general result (2.10).

\section{Applications}

We will now give a brief outline of some applications of the theory described in Section 2 to rheological models, in order to show its capability of treating several types of microrheological models at various levels of description. We will omit most of the details and merely present the most important results. For further information about the rheological models we refer to the review paper [8]. 


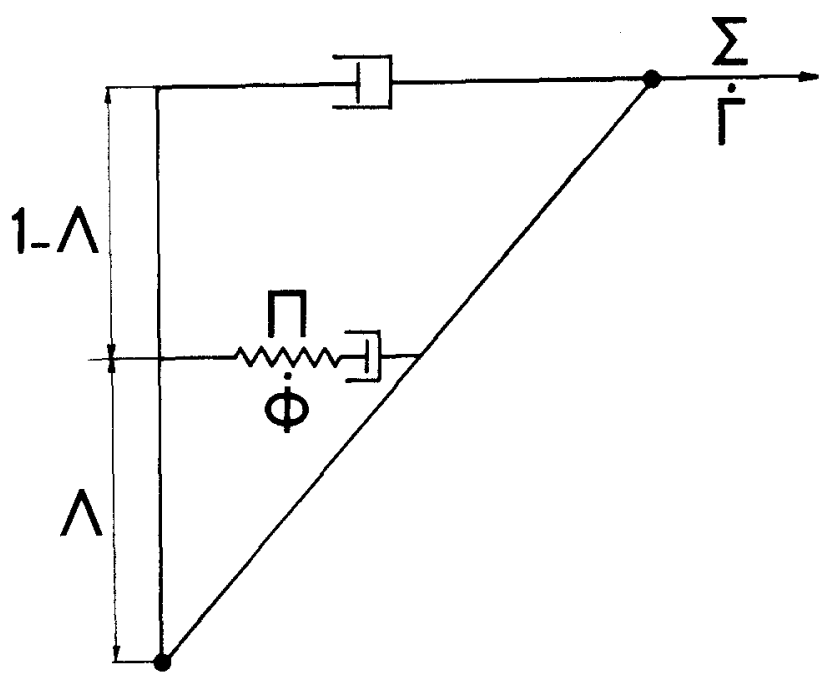

Fig. 2. The triangle model. In this construction the external force $\Sigma$ is split into a dissipation part $\Sigma^{D}$ and a reversible part $\Sigma^{R}=\Lambda^{T} \cdot \Pi$ which is different from the thermodynamic force $\Pi$.

First we consider the 'elastic dumbbell model'. In this model, which is used for polymeric liquids, a polymer molecule is represented by a dumbbell consisting of two beads connected by a spring immersed in a viscous fluid.

The relevant equations for this model are [1] the stress tensor expression

$$
T=2 \eta D+n k T \int \psi \frac{\partial}{\partial q}\left(\ln \frac{\psi}{\psi_{0}}\right) q \mathrm{~d} q^{3}
$$

and the diffusion equation

$$
\frac{\partial \psi}{\partial t}=-\frac{\partial}{\partial q} \cdot(\psi L \cdot q)+\frac{2 k T}{\zeta} \frac{\partial}{\partial q} \cdot\left(\psi \frac{\partial}{\partial q} \ln \frac{\psi}{\psi_{0}}\right)
$$

In these equations $\psi=\psi(q, t)$ is the configuration distribution function, $\psi_{0}$ the equilibrium distribution, $q$ the dumbbell vector, $L$ the velocity gradient tensor, $D=\frac{1}{2}\left(L+L^{T}\right)$ the rate-of-strain tensor, $T$ the stress tensor, $n$ the number density of dumbbells, $k$ Boltzmann's constant, $T$ the temperature, $\eta$ the fluid viscosity and $\zeta$ a friction modulus.

It turns out that the equations (3.1) and (3.2) can be brought into the form (2.10) by introducing the free energy functional [2]

$$
A\{\psi\}=n k T \int \psi \ln \frac{\psi}{\psi_{0}} \mathrm{~d} q^{3}
$$


and the corresponding thermodynamic force

$$
\mu=\frac{\delta A}{\delta \psi}=n k T\left(1+\ln \frac{\psi}{\psi_{0}}\right)
$$

The matrix representation then becomes

$$
\left[\begin{array}{c}
T \\
\frac{\partial \psi}{\partial t}
\end{array}\right]=\left[\begin{array}{ll}
2 \eta \mathbf{I}: & \frac{\partial}{\partial q}(\psi q) * \\
-\frac{\partial}{\partial q}(\psi q): & \frac{-2}{n \zeta} \frac{\partial}{\partial q} \cdot \psi \frac{\partial}{\partial q}
\end{array}\right]\left[\begin{array}{r}
L \\
-\mu
\end{array}\right]
$$

with $I_{i j k m}=\frac{1}{2}\left(\delta_{i k} \delta_{m j}+\delta_{i m} \delta_{k j}\right)$ and $(\cdots) *(\cdots) \equiv \int(\cdots)(\cdots) \mathrm{d}^{3} q$.

As has been mentioned already in the introduction, in many cases a description is possible at various levels of description. In the present case we just have considered the level based upon the distribution function $\psi$. A next higher level is based upon the configuration tensor $S=\langle q q\rangle$ (the brackets denote an average with respect to $\psi$ ). In that case we have instead of (3.1) and (3.2):

$$
T=-n k T 1+2 \eta D+n \kappa S,
$$

in which $\kappa$ is the spring constant, and

$$
\stackrel{\nabla}{S}=\frac{4 k T}{\zeta} 1-\frac{4 \kappa}{\zeta} S
$$

with $\stackrel{\nabla}{S}=\dot{S}-L \cdot S-S \cdot L^{T}$ the so-called upper-convected derivative of $S$. These equations may be written in the matrix form (2.10) by introducing the free energy function [6]

$$
A=1 / 2 n k T \log (\operatorname{det} S)+1 / 2 n k \operatorname{tr} S
$$

with the associated thermodynamic force

$$
M=\frac{\partial A}{\partial S}=1 / 2 n \kappa\left(1-\frac{k T}{\kappa} S^{-1}\right)
$$

The matrix representation then becomes

$$
\left[\begin{array}{l}
T \\
\dot{S}
\end{array}\right]=\left[\begin{array}{cc}
2 \boldsymbol{\eta} & -\boldsymbol{\Lambda}^{T} \\
\boldsymbol{\Lambda} & \boldsymbol{\beta}
\end{array}\right]\left[\begin{array}{c}
L \\
-M
\end{array}\right],
$$


with

$$
\Lambda_{i j k m}=S_{i k} \delta_{m j}+S_{i m} \delta_{j k}, \eta=\eta \mathbf{I} \quad \text { and } \quad \beta=\frac{4}{n \zeta} \Lambda .
$$

This equation may be generalized by modifying the expressions for $M(S)$ and $\boldsymbol{\beta}$. The so-called Giesekusmodel $[3,4]$ for instance, may prove to correspond to the case $\boldsymbol{\beta}=B \cdot \boldsymbol{\Lambda}$ and $M=1 / 2 \mu\left(l-S^{-1}\right)$ with $B$ a 'generalized mobility tensor' and $\mu$ an elasticity-modulus.

From the $\psi$-level one may also go to a lower level of description, by considering the forces and mean fluxes at a point in configuration space, we have the balance of forces (see also Fig. 3):

$$
f^{H}-k T \frac{\partial}{\partial q} \ln \psi+k T \frac{\partial}{\partial q} \ln \psi_{0}=0
$$

or

$$
f^{H}+f^{B}-f^{I}=0
$$

in which $\dot{d}=L \cdot q$, the relative fluid velocity at the two beads, $f^{H}=1 / 2 \zeta(\dot{d}-\dot{q})$, the hydrodynamic force, $f^{B}$ the so-called Brownian force and $f^{I}$ the spring force (see [1], p. 15). For the flux $\dot{q}$ we also have

$$
\dot{q}=\dot{d}-\frac{2 k T}{\zeta} \frac{\partial}{\partial q} \ln \frac{\psi}{\psi_{0}}
$$

These equations may be expressed in the form (2.10) by introducing a local free energy in configuration space:

$$
a=k T \ln \frac{\psi}{\psi_{0}}
$$

and the associated thermodynamic force

$$
m=\frac{\partial a}{\partial q}=-f^{B}+f^{I}
$$

The matrix formulation then becomes

$$
\left[\begin{array}{c}
f^{H} \\
\dot{q}
\end{array}\right]=\left[\begin{array}{ll}
0 & -I \\
1 & \frac{2}{\zeta} I
\end{array}\right]\left[\begin{array}{r}
\dot{d} \\
-m
\end{array}\right] .
$$


In the case of a rigid dumbbell the force $f^{I}$ is a constraining force, determined by the hydrodynamic forces on the beads and the thermodynamic force $m$ only consists of a Brownian force, which in this case is always perpendicular to $q$. This may be expressed by using the projection operator $P=(I-e e)$ with $e=q /|q|$. Instead of (3.15) one then obtains

$$
\left[\begin{array}{c}
f^{H} \\
\dot{q}
\end{array}\right]=\left[\begin{array}{ll}
\frac{\zeta}{2}(1-P) & -P \\
P & \frac{2}{\zeta} P
\end{array}\right]\left[\begin{array}{c}
\dot{d} \\
-m
\end{array}\right] .
$$

In Fig. 4 the equilibrium of forces is sketched. Note that in this case there is no longer a direct coupling between the hydrodynamic force $f^{H}$ and the thermodynamic force $m$. This is also clear from the expression (3.16).

We have illustrated some applications of the present theory to the dumbbell model. The theory may also be applied to other rheological models like reptation theories and transient network models. Some examples are given in [8], other applications will be published elsewhere.

\section{Discussion}

The general theory, presented in Section 2 was applied in Section 3 to the elastic dumbbell model and some related models. The important steps in these applications were: the selection of a level of description, and the corresponding system variables

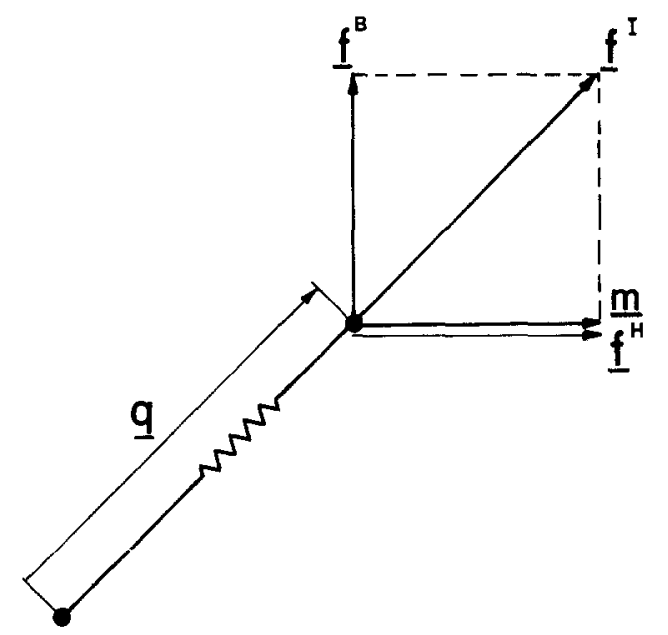

Fig. 3. Equilibrium of forces in the elastic dumb-bell model. 


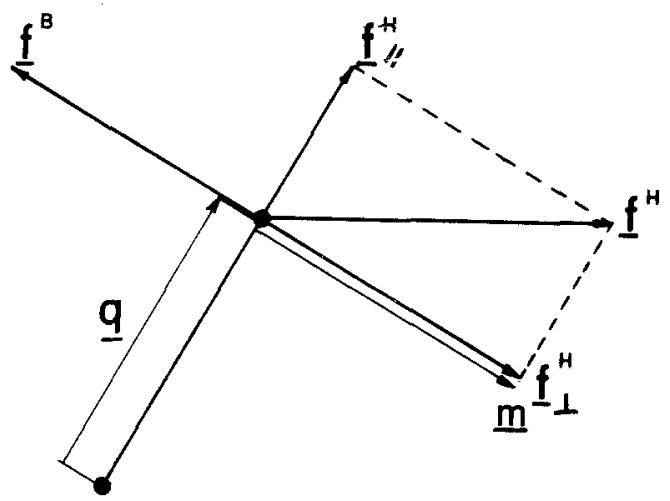

Fig. 4. Equilibrium of forces in the rigid dumb-bell model.

$(\Phi, \Sigma, \dot{\Gamma})$ and the determination of the free energy function (functional) $A(\Phi)$ with the associated thermodynamic force $\Pi(\Phi)$. From the evolution equations and the stress tensor expressions (or - at a lower level of description - the balance of forces) the matrix elements $\eta, \beta$, and $\Lambda$ of the expression (2.10) are then obtained. In applications to systems for which a rheological model is not yet available the same method may be used in order to construct such a model. The advantage of this approach is that a clear distinction is made between some isolated parts of the problem: the choice of a level of description, the free energy function, the dissipativa $(\eta$ and $\beta$ ) and reversible $(\Lambda)$ couplings etc., while the consistency of the whole model remains assured.

\section{Appendix A}

In this appendix the derivation of the equations (2.9) will be discussed. We first consider the case of sealar variables. The dissipation form (2.6) then becomes

$$
\Delta=A \gamma+p B
$$

in which $A, \gamma, p$ and $B$ are sealar quantities corresponding to $\Sigma, \dot{\Gamma},-\Pi$ and $\dot{\Phi}$ of (2.6), respectively. So $\gamma$ has an odd and $p$ and even parity with respect to 'macroscopic time reversal'. We decompose $A$ and $B$ in their even and odd parts:

$$
A=a+\alpha, \quad B=b+\beta,
$$

with $a=A^{+}, b=B^{+}, \alpha=A^{-}, \beta=B^{-}$. From (2.7) and (2.8) we then obtain:

$$
\begin{aligned}
& \alpha \gamma+p b \geqslant 0 \\
& a \gamma+p \beta=0 .
\end{aligned}
$$


The constitutive relations between the quantities $A, B, \gamma$ and $p$ should be formulated in such a way that the equations (A.3) and (A.4) are obeyed for arbitrary processes compatible with the constitutive equations. We take $\gamma$ and $p$ as independent variables and consider constitutive equations which may be expressed in matrix form. So from (A.3) we have

$$
\left[\begin{array}{l}
\alpha \\
b
\end{array}\right]=\left[\begin{array}{ll}
x & u \\
v & y
\end{array}\right]\left[\begin{array}{l}
\gamma \\
p
\end{array}\right] .
$$

The inequality (A.3) then becomes

$$
x \gamma^{2}+y p^{2}+(u+v) p \gamma \geqslant 0 .
$$

We will only consider here the case of matrix elements which are independent of $\gamma$. Then, all matrix elements in (A.5) are even quantities and from the parity of the other variables in (A.5) it follows that $u=v=0$. So from (A.6) we have $x \geqslant 0, y \geqslant 0$ and

$$
\left[\begin{array}{l}
\alpha \\
b
\end{array}\right]=\left[\begin{array}{ll}
x & 0 \\
0 & y
\end{array}\right]\left[\begin{array}{l}
\gamma \\
p
\end{array}\right] .
$$

We now return to the equation (A.4). The corresponding matrix expression analogues to (A.5) becomes

$$
\left[\begin{array}{l}
a \\
\beta
\end{array}\right]=\left[\begin{array}{ll}
x^{\prime} & u^{\prime} \\
v^{\prime} & y^{\prime}
\end{array}\right]\left[\begin{array}{l}
\gamma \\
p
\end{array}\right] .
$$

Then, from (A.4) we obtain

$$
x^{\prime} \gamma^{2}+y^{\prime} p^{2}+\left(u^{\prime}+v^{\prime}\right) p \gamma=0
$$

Again, we assume no dependence of the matrix elements upon $\gamma$, so we obtain $x^{\prime}=0$ and $y^{\prime}=0$ and since (A.9) has to be obeyed for arbitrary $\gamma$ and $p$ we have: $u^{\prime}=-v^{\prime}$. So (A.8) becomes

$$
\left[\begin{array}{l}
a \\
\beta
\end{array}\right]=\left[\begin{array}{rr}
0 & -Z \\
Z & 0
\end{array}\right]\left[\begin{array}{l}
\gamma \\
p
\end{array}\right],
$$

with $Z=v^{\prime}$. From (A.2), (A.8) and (A.10) we finally obtain

$$
\left[\begin{array}{l}
A \\
B
\end{array}\right]=\left[\begin{array}{rr}
X & -Z \\
Z & Y
\end{array}\right]\left[\begin{array}{l}
\gamma \\
p
\end{array}\right],
$$


in which $X=x, Y=y$. This completes the proof of expression (2.10) for the case of scalar variables.

Note that in the derivation we have assumed that all matrix elements were constants. Since, however $p$ is an even quantity $r$ dependence upon $p$ - or any other thermodynamic variable of state - will not invalidate our results. A dependence upon $\gamma$, however will change the conclusions derived from (A.4). This will not be discussed here further. So we will restrict ourselves to systems in which the matrix elements in the final expression (A.11) may depend upon thermodynamic variables at state but are independent of $\gamma$.

Finally we will discuss some aspects of the general case in which arbitrary tensioral or even functional quantities arise.

In general, the dissipation equation then becomes (cf. (2.6))

$$
\Delta=\Sigma \cdot \dot{\Gamma}-\Pi * \dot{\Phi}
$$

The and the $*$ in this expression denote the appropriate inner products in the spaces in which $\Sigma, \dot{\Gamma}$, respectively $\Pi, \dot{\Phi}$ are defined. (Contrary to (2.6) we now explicitly distinguish two types of inner products.) Just as in the case of Cartesian tensors, for which the "' operator in $v=A \cdot b$ in which $A$ is a second-order-tensor, corresponds to the operator ' ' in the inner product $\phi=a \cdot b$ we use the inner product operators in (A.12) also in matrix expressions like (2.10). So, corresponding to (A.12) we write

$$
\left[\begin{array}{c}
\Sigma \\
\dot{\Phi}
\end{array}\right]=\left[\begin{array}{cc}
\eta & -\Lambda^{T} * \\
\Lambda \cdot & \beta *
\end{array}\right]\left[\begin{array}{c}
\dot{\Gamma} \\
-\Pi
\end{array}\right] .
$$

(In some cases (like equation (3.5)) operators '.' or ' *' may be expressed in a different form.) The reason that the transposed (or in general the adjoint) $\Lambda^{T}$ occurs in this expression is that in a derivation similar to that of (A.9) one obtains an identity of the type

$$
(U * \Gamma) \cdot \dot{\Gamma}+(V \cdot \dot{\Gamma}) * V=0
$$

in which $U$ and $V$ correspond to $u^{\prime}$ and $v^{\prime}$ in (A.9). On using the definition of the transposed (adjoint) $U^{T}$ of $U$ one obtains

$$
\left(U^{T} \cdot \dot{\Gamma}\right) * \Gamma+(V \cdot \dot{\Gamma}) * \Gamma=0
$$

so $V=-U^{T} \equiv \Lambda$, in accordance with $(2.10)$. 


\section{References}

1. Bird, R.B., Armstrong, R.L. and Hassager, O., Dynamics of Polymeric Vol. 1, New York: Wiley (1987).

2. Dui, M., J. Chem. Phys. 79 (1983) 5080.

3. Giesekus, H., Rhed. Acta 2 (1962) 50-62.

4. Giesekus, H.J., Non Newtonian Fluid Mech. 11 (1982) 69-109.

5. Grmela, M., Physica 21D (1986) 179-212.

6. Grmela, M. and Carreau, P.J., J. Non Newtonian Fluid Mech. 23 (1987) 271-294.

7. de Groot, S.R. and Mazur, P., Non-equilibrium Thermodynamics, Amsterdam: North-Holland (1969).

8. Jongschaap, R.J.J., Rep. Progr. Phys. 53 (1990) 1.

9. Lodge, A.S., Trans. Faraday Soc. 52 (1956) 120-130. 